\title{
Formulation of Oil-in-Water Cream from \\ Mangosteen (Garcinia mangostana L.) Pericarp Extract Preserved by Gamma Irradiation
}

\author{
E. Isabella ${ }^{*}$ and T. Pohan \\ Faculty of Pharmacy, Pancasila University, Srengseng Sawah, Jagakarsa, Jakarta 12640, Indonesia
}

\section{ARTICLE INFO}

Article history:

Received 24 November 2013

Received in revised form 20 December 2013

Accepted 26 December 2013

Keywords:

Anti-aging cream

Mangosteen pericarp extract

Garcinia mangostana L.

Decontamination

Gamma irradiation

\begin{abstract}
A B S T R A C T
The aim of this research was to formulate mangosteen (Garcinia mangostana L.) pericarp extract into oil-in-water $(\mathrm{O} / \mathrm{W})$ cream which stable and durable. In order to improve the shelf life durability of the formula, the irradiation of formula using gamma rays from cobalt- 60 was done. The concentrations of extract were varied to 1,2 and $3 \%$ and were irradiated by gamma rays with doses of 5, 7.5 and $10 \mathrm{kGy}$. Physicochemical and microbiological properties of these formulas were carried out for 90 days stored at $30 \pm 2{ }^{\circ} \mathrm{C}$ and $40 \pm 2{ }^{\circ} \mathrm{C}$. It was found that both of physicochemical and microbiological properties of the formulas were changed by extract concentrations and after irradiation. The irradiated formulas with dose up to $7.5 \mathrm{kGy}$, which were stored at $30 \pm 2{ }^{\circ} \mathrm{C}$ and $40 \pm 2{ }^{\circ} \mathrm{C}$, presented acceptable physicochemical and microbiological stability for at least 90 days. Analysis by TLC (Thin Layer Chromatography)-densitometry to study decomposition of G. mangostana pericarp extract cream formulas was carried out $24 \mathrm{~h}$ after preparation and 90 day after storage. The result of TLC-densitometry analysis showed that $G$. mangostana pericarp extract in the cream formulas did not develope significant decomposition after 90 days of storage. Decontamination dose for all formulas was found to be about $7.5 \mathrm{kGy}$. At this condition, the bacteria and moldyeast have been killed, without reducing the antioxidant activity.
\end{abstract}

(C) 2013 Atom Indonesia . All rights reserved

\section{INTRODUCTION}

Skin is subject to deterioration through dermatological disorders, environmental abuse, or through the normal aging process (chronoaging), which may be accelerated by exposure of skin to sun (photoaging). Environmental abuse such as ultraviolet radiation, air pollutants, chemicals, air conditioning, central heating, alcohols, daily stress and other environmental factors can result in the generation of free radicals in the exposed skin which can lead to premature aging of skin [1]. The visible signs of aging skin are wrinkles, lines, sagging, hyperpigmentation and age spots [2,3].

The effective treatment and prevention of skin conditions due to natural or environmental aging may be obtained through the application of cosmetic compositions to the skin which comprises collagen stimulators, phytoestrogens, vitamins, allantoin, placental extract, antioxidants [2], some of which, such as vitamins and antioxidants can be found in many plants. One type of plants that has potential antioxidant activity is mangosteen.

\footnotetext{
* Corresponding author.

E-mail address: lebelleanore@gmail.com
}

Mangosteen (Garcinia mangostana L.) from family Clusiaceae, is a tropical fruit from Southeast Asian. People have used the pericarp of $G$. mangostana as a traditional medicine for the treatment of abdominal pain, diarrhea, dysentery, infected wound and chronic ulcer [4]. Moreover, recent scientific studies reported that pericarp of G. mangostana is a source of xanthones and other bioactive subtances which possessed several biological and pharmacological properties such as antioxidant, antiproliferative, antihistamine, antifungal, antibacterial and induction of apoptosis in cancer cell line [5-7]. In addition, Ochiai et al. [8] reported that G. mangostana pericarp extract have bioactive subtances which inhibit matrix metalloproteinases, that are well known to be zinccontaining proteolytic enzymes that hydrolyze extracellular matrix-forming protein including collagen and to cause changes due to the aging of the skin.

The skin treatment composition of this research is preferably on oil-in-water $(\mathrm{O} / \mathrm{W})$ type emulsion since this type of emulsion affords better cosmetic feel to the product, light when used, easily washed and leave no greasy feeling [3]. Topically applied formulas must not contain microbials 
exceeding the permissible limits. According to The National Agency of Drugs and Food Controls of Indonesia (NADFC = BPOM) [9], the maximum contamination limit of cosmetics for microbes and fungal is $10^{3} \mathrm{cfu} / \mathrm{g}$, respectively. Cobalt-60 source, which is commonly used for gamma irradiation, can be used for sterilization/decontamination of cosmetic raw materials and finished products. Aiming at the reduction in microbiological content, the method cannot make something radioactive or leave any residual radioactive and also can be processed at room temperature. Gamma irradiation can penetrate the packaging materials and sealed packages containing the final product, thus destroying the existing microorganisms and extend the shelf life of products $[10,11]$. The objective of this research was to develop anti-aging cream from $G$. mangostana crude extract and to extend shelf life of formulas by gamma irradiation.

\section{EXPERIMENTAL METHODS}

\section{Materials and equipments}

Garcinia mangostana fruits were purchased from a local plantation in Leuwiliang, Bogor, West Java. The authentication of the plant material was carried out at the Herbarium Bogoriensis Research Center for Biology, Indonesian Institute of Sciences, Cibinong, West Java.

Stearic acid, cetyl alcohol (1-hexadecanol), isopropyl myristate, glyceryl monostearate, propylene glycol, triethanolamine, and perfume were purchased from Tritunggal Arthamakmur; butylated hydroxytoluene, methylparaben, propylparaben, and distilled water were purchased from Brataco. DPPH (1,1-diphenyl-2picrylhydrazyl) radical, $70 \%$ ethanol, cerium sulfate, methanol, chloroform and $\mathrm{GF}_{254}$ silica plate were used in this research.

Equipments used were gamma irradiator "Karet Alam" with cobalt-60 source (activity: $120 \mathrm{kCi}$ at 2012), vacuumized rotary evaporator (Büchi), densitometer (CAMAG), UV light cabinet (CAMAG), spectrophotometer UV-VIS (Shimadzu UV 1800), laminar air flow, oven (Memmert), viscometer (Brookfield RV), waterbath (Memmert), analytical balance (Mettler Toledo AB 204), microscope (Olympus Optical), stirrer (Eurostar), centrifuge (Porta), extensometer, $\mathrm{pH}$ meter (Meterohm type 620), ultrasonicator, hot plate, chromatograph chamber, incubator, objective glass, thermometer (Assistant).

\section{Crude extract formulation}

The pericarp of $G$. mangostana fresh fruits were chopped into small pieces, dried and extracted with $70 \%$ ethanol for one weeks, four times, at room temperature. The filtrates were pooled and concentrated by a vacuumized rotary evaporator at $40^{\circ} \mathrm{C}$.

\section{O/W cream from G. mangostana pericarp extract formulation}

Stearic acid, cetyl alcohol, isopropyl myristate, glyceryl monostearate and butylated hydroxytoluene were mixed, molten under heating and maintained at $70^{\circ} \mathrm{C}$ (dispersed phase). The other ingredients were mixed, dissolved under heating and maintained at $70^{\circ} \mathrm{C}$ (dispersing phase). The dispersed phase was gradually added to the dispersing phase at $70^{\circ} \mathrm{C}$ with sufficient stirring, G. mangostana pericarp extract and perfume were mixed into the base at $40^{\circ} \mathrm{C}$. It was then emulsified to homogeneity by stirring (15 min, $150 \mathrm{rpm} . \mathrm{min}-1)$.

Table 1. Composition $(\% \mathrm{w} / \mathrm{w})$ of $\mathrm{O} / \mathrm{W}$ cream from G. mangostana pericarp extract.

\begin{tabular}{lccc}
\hline \multirow{2}{*}{ Components } & \multicolumn{3}{c}{ Formula code } \\
\cline { 2 - 4 } & $\mathrm{A}$ & $\mathrm{B}$ & $\mathrm{C}$ \\
\hline G. mangostana pericarp extract & 1 & 2 & 3 \\
stearic acid & 5 & 5 & 5 \\
cetyl alcohol & 2.75 & 2.75 & 2.75 \\
isopropyl myristate & 10 & 10 & 10 \\
glyceryl monostearate & 3 & 3 & 3 \\
propylene glycol & 10 & 10 & 10 \\
triethanolamine & 1 & 1 & 1 \\
butylated hydroxytoluene & 0.05 & 0.05 & 0.05 \\
methylparaben & 0.15 & 0.15 & 0.15 \\
propylparaben & 0.05 & 0.05 & 0.05 \\
perfume (musk) & 1 & 1 & 1 \\
distilled water & 66 & 65 & 64 \\
\hline
\end{tabular}

\section{Irradiation process}

Irradiation was performed at room temperature using cobalt-60 source from Gamma Irradiator "Karet Alam". All samples were irradiated at doses of 5, 7.5 and $10 \mathrm{kGy}$. The samples were G. mangostana pericarp extract and cream formulas (code: A, B, C).

Organoleptic properties, $\mathrm{pH}$, DPPH radical scavenging activities and TLC profiles were carried out for extract samples, while physicochemical properties (organoleptic, emulsion type, centrifuge, spreadability, viscosity and flow properties, mean particle size and $\mathrm{pH}$ ), microbiological (total plate count) properties and TLC densitometry profiles were carried out for cream formulas at room 
temperature $\left(30 \pm 2^{\circ} \mathrm{C}\right)$ and high temperature $\left(40 \pm 2^{\circ} \mathrm{C}\right)$.

Unirradiated formulas were used as controls to detect the changes resulting from the action of ionizing radiation on the formulas investigated.

\section{DPPH radical scavening activities}

The decrease of the absorption at $517 \mathrm{~nm}$ of the DPPH solution after addition of the G. mangostana extract samples (unirradiated and irradiated) were measured in a glass cuvette (1 cm length). Each of an aliquot $(1 \mathrm{~mL})$ of $0.4 \mathrm{mM}$ methanolic DPPH solution was added to 10 , $20,30,40$ and $50 \mu \mathrm{L}$ of $1000 \mathrm{mg} / \mathrm{L}$ methanolic $G$. mangostana extract samples solution so that the concentrations in the cuvette were $2,4,6,8$ and $10 \mathrm{mg} / \mathrm{L}$. All the tests were performed in triplicate at $37^{\circ} \mathrm{C}$ (incubation). The absorption was monitored at $30 \mathrm{~min}$ after incubation. Absorbance values were corrected for radical decay using blank solutions. Reduction of DPPH was followed by monitoring the decrease in absorbance at $517 \mathrm{~nm}$ until the reaction reached a plateau. The inhibition concentration $\left(\mathrm{IC}_{50}\right)$ is the amount of antioxidant necessary to decrease the initial DPPH by $50 \%$. The lower the $\mathrm{IC}_{50}$ is, the higher the antioxidant activity.

\section{TLC profiles of G. mangostana pericarp extract}

Unirradiated and irradiated extract solutions $[1 \%(\mathrm{w} / \mathrm{v})]$ were prepared and analyzed by TLC using chloroform - methanol (12:1) as eluent. The spots were observed and marked under UV 254 $\mathrm{nm}$ light cabinet, then sprayed with $1 \%$ cerium sulfate reagent in $10 \%$ sulfuric acid and dried on the hot plate to form visible spots.

\section{Physicochemical properties}

All test were performed on unirradiated and irradiated cream formulas at doses of 5, 7.5, and 10 kGy. The results were analyzed statistically: (1) Organoleptic properties: formulas were evaluated by their color, odor and homogeneity; (2) Emulsion type: formulas were mixed with sudan III on the objective glass and investigated by light microscope; (3) Centrifuge: formulas were placed in centrifuge tubes, vigorously shaken for $15 \mathrm{~min}$ at $2500 \mathrm{rpm} \cdot \mathrm{min}^{-1}$ and observed whether there was a separate section or not; (4) Spreadability: formulas were measured using extensometer; (5) Viscosity and flow properties: formulas were measured by Brookfield rheometer (RV type); (6) Mean particle size: particle sizes of formulas were investigated by light microscope. Results were in an average; (7) $\mathrm{pH}$ : formulas were measured by $\mathrm{pHmeter}$.

\section{Microbiological properties}

Number of total bacteria and total mold-yeast of the cream formulas were determined by plate count method according to SNI 01-2897-1992 [12]. A total of $10 \mathrm{~g}$ cream formulas were added into $90 \mathrm{~mL}$ of buffer phosphate solution ( $\mathrm{pH} 7.2)$, thus obtained the dilution of $1: 10$, homogenized, then gradually diluted to $10^{-2}, 10^{-3}, 10^{-4}, 10^{-5}$ and $10^{-6}$. Each of a total of $1 \mathrm{~mL}$ of diluted sample was poured into a sterile Petri dish, 15-20 mL of nutrient agar medium was added, shaken gently until evenly mixed sample, silenced until frozen, then the Petri dish was reversed and incubated at $30-35^{\circ} \mathrm{C}$ for $48 \mathrm{~h}$. Similar as described in the determination number of total bacteria, each of a total of $1 \mathrm{~mL}$ of diluted sample was poured into a sterile Petri dish, $15-20 \mathrm{~mL}$ of potato dextrose agar medium was added, shaken gently until evenly mixed sample, silenced until frozen, then the Petri dish was reversed and incubated at $20-24^{\circ} \mathrm{C}$ for 5 days. The number of total bacteria and total mold-yeast were calculated using the following equation:

$$
N=\frac{\Sigma C}{(p+0.1 q+0.01 r+\cdots) d}
$$

where $N$ is total plate count (cfu/g); $\Sigma \mathrm{C}$ is total colony of all counted Petri dish; $p, q, r$ are total counted Petri dish at first, second, third dilution; $d$ is first counted dilution.

\section{TLC-densitometry}

G. mangostana pericarp extract was separated from cream formulas and identified by TLCdensitometry. The results were compared between unirradiated and irradiated formulas. Each of a total of $100 \mathrm{mg}$ of formula was dissolved in methanol $(10000 \mathrm{mg} / \mathrm{L})$, then $20 \mu \mathrm{L}$ of $10000 \mathrm{mg} / \mathrm{L}$ formula solution was spotted on $\mathrm{GF}_{254}$ silica plate and analyzed by TLC using chloroform - methanol (12:1) as eluent. The spots were observed by densitometer at $317 \mathrm{~nm}$. 


\section{Statistical analysis}

Physicochemical properties (spreadability, viscosity, mean particle size, $\mathrm{pH}$ ) and microbiological (total plate count) test results were given as the mean of 3 experiments and ANOVA two ways $(\mathrm{p}=0.05)$ from Minitab 16 software program was employed for analyses.

\section{RESULTS AND DISCUSSION}

\section{Mangosteen pericarp extract}

Extraction of $4.5 \mathrm{~kg}$ of $G$. mangostana pericarps, got $19.32 \%$ of crude extract. Physicochemical properties of unirradiated and irradiated G. mangostana pericarp extract were summarized in Table 2. It was determined that there was no change in the physicochemical properties of irradiated $G$. mangostana pericarp extract at 3 different doses.

Table 2. Physicochemical properties and $\mathrm{IC}_{50}$ values of G. mangostana pericarp extract before and after irradiation.

\begin{tabular}{ccccc}
\hline \multirow{2}{*}{ Properties } & \multirow{2}{*}{ Unirradiated } & \multicolumn{4}{c}{ Irradiated $(\mathrm{kGy})$} \\
\cline { 3 - 5 } & & 5 & 7.5 & 10 \\
\hline \multirow{2}{*}{ Organoleptic } & $\begin{array}{c}\text { viscous, dark brown, } \\
\text { characteristic }\end{array}$ & + & + & + \\
$\mathrm{pH}$ & 5.45 & 5.55 & 5.55 & 5.60 \\
$\mathrm{IC}_{50}(\mathrm{mg} / \mathrm{L})$ & 8.08 & 6.87 & 5.30 & 4.71 \\
\hline$*(+:$ no change; - : change $)$ & & & &
\end{tabular}

The antioxidant activity of $G$. mangostana pericarp extract which was implemented in linear regression:

$$
y=a x+b
$$

between extract concentration versus percent of inhibition were shown in Fig. 1. The inhibition concentration-fifty $\left(\mathrm{IC}_{50}\right)$ which expresses the ability of the extract to inhibit $50 \%$ of DPPH radical calculated by substituting $\mathrm{Y}$ by 50 for each linear regression curve, in order to obtain the $\mathrm{IC}_{50}$ values.

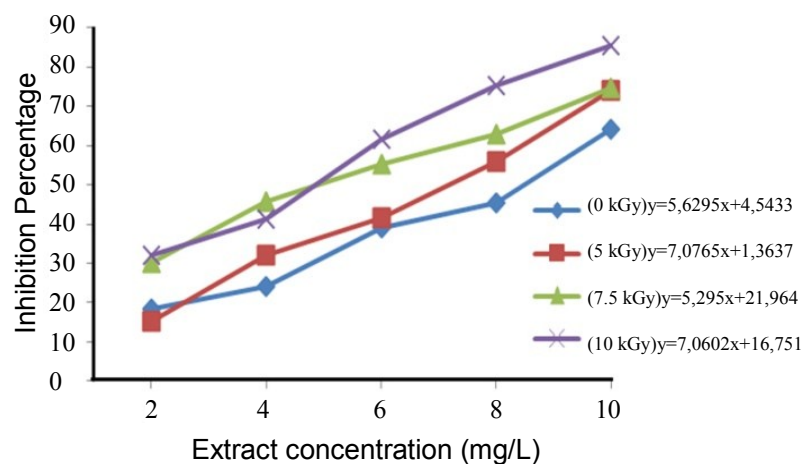

Fig. 1. Curve of extract concentration versus inhibition percentage.
Table 2 shows that the increase in radiation dose until $10 \mathrm{kGy}$ caused the decrease in the $\mathrm{IC}_{50}$ value, which means that the antioxidant activity increased. The extract is declared to have a very strong antioxidant activity if the $\mathrm{IC}_{50}$ value $<50 \mathrm{mg} / \mathrm{L}$ [13]. This fact suggested that the irradiation dose until $10 \mathrm{kGy}$ is still feasible for the microbial decontamination of $G$. mangostana pericarp extract.

Based on the TLC profiles there were at least 3 spots observed in extract formulations (Fig. 2). It was showed that the irradiated spots at the 3 different doses were not significantly different from the unirradiated spots. The irradiation dose until $10 \mathrm{kGy}$ did not cause any significant changes in TLC profiles of G. mangostana pericarp extract.

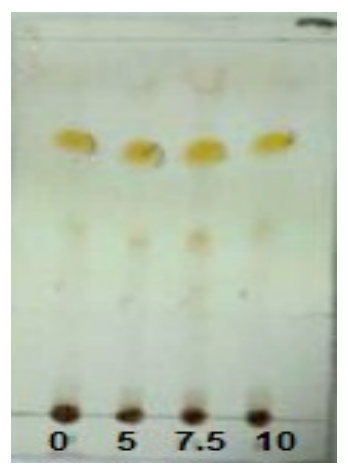

Fig. 2. TLC of unirradiated and irradiated G. mangostana pericarp extract (condition: silica gel $\mathrm{GF}_{254}$, chloroform methanol (12:1), UV light at $254 \mathrm{~nm}, 1 \%$ cerium sulphate in $10 \%$ sulfuric acid).

\section{Physicochemical properties}

Organoleptic properties, emulsion type and centrifuge (physical properties) of unirradiated and irradiated formulas were summarized in Table 3. Physical properties of formulas stored at room and high temperature did not suffer perceptible alterations during the study period of 90 days. The increasing extract concentrations and irradiation doses affected its color parameter unsignificantly.

Spreadability value of unirradiated and irradiated formulas were summarized in Table 4. It was determined that increasing extract concentrations caused increasing spread ability values, while increasing doses of irradiation caused decreasing spread ability values as shown in Fig. 3. Changes in spreadability value of all formulas seem depends on extract concentrations $(p<0.05)$ and irradiation dose levels $(\mathrm{p}<0.05)$. Apparent spreadability of formulas resulted in increased values when stored at high temperature and during 90 days of storage. 
Table 3. Physical properties of formulas before and after irradiation evaluated after $24 \mathrm{~h}$ of formulation and at $90^{\text {th }}$ day of analyses.

\begin{tabular}{|c|c|c|c|c|c|c|c|c|c|c|c|c|c|}
\hline \multirow{4}{*}{$\begin{array}{l}\mathrm{C} \\
\mathrm{o} \\
\mathrm{d} \\
\mathrm{e}\end{array}$} & \multirow{4}{*}{$\begin{array}{l}\text { Storage } \\
\text { conditions }\end{array}$} & \multicolumn{6}{|c|}{$24 \mathrm{~h}$ after formulation } & \multicolumn{6}{|c|}{$90^{\text {th }}$ day of analyses } \\
\hline & & \multirow{2}{*}{\multicolumn{3}{|c|}{ Unirradiated }} & \multicolumn{3}{|c|}{ Dose (kGy) } & \multirow{2}{*}{\multicolumn{3}{|c|}{ Unirradiated }} & \multicolumn{3}{|c|}{ Dose (kGy) } \\
\hline & & & & & \multirow{2}{*}{5} & \multirow{2}{*}{7.5} & \multirow{2}{*}{10} & & & & \multirow{2}{*}{5} & \multirow{2}{*}{7.5} & \multirow{2}{*}{10} \\
\hline & & $\mathrm{O}$ & ET & $\mathrm{C}$ & & & & $\mathrm{O}$ & ET & $\mathrm{C}$ & & & \\
\hline A & \multirow{3}{*}{$\begin{array}{c}\text { Room } \\
\text { temperature } \\
30 \pm 2^{\circ} \mathrm{C}\end{array}$} & LB, M, H & $\mathrm{O} / \mathrm{W}$ & $\mathrm{x}$ & + & + & + & $\mathrm{LB}, \mathrm{M}, \mathrm{H}$ & $\mathrm{O} / \mathrm{W}$ & $\mathrm{x}$ & + & + & + \\
\hline B & & $\mathrm{DB}, \mathrm{M}, \mathrm{H}$ & $\mathrm{O} / \mathrm{W}$ & $\mathrm{x}$ & + & + & + & $\mathrm{DB}, \mathrm{M}, \mathrm{H}$ & $\mathrm{O} / \mathrm{W}$ & $\mathrm{x}$ & + & + & + \\
\hline $\mathrm{C}$ & & $\mathrm{DB}, \mathrm{M}, \mathrm{H}$ & $\mathrm{O} / \mathrm{W}$ & $\mathrm{x}$ & + & + & + & $\mathrm{DB}, \mathrm{M}, \mathrm{H}$ & $\mathrm{O} / \mathrm{W}$ & $\mathrm{x}$ & + & + & + \\
\hline A & \multirow{3}{*}{$\begin{array}{c}\text { High } \\
\text { temperature } \\
40 \pm 2^{\circ} \mathrm{C}\end{array}$} & $\mathrm{LB}, \mathrm{M}, \mathrm{H}$ & $\mathrm{O} / \mathrm{W}$ & $\mathrm{x}$ & + & + & + & $\mathrm{LB}, \mathrm{M}, \mathrm{H}$ & $\mathrm{O} / \mathrm{W}$ & $\mathrm{x}$ & + & + & + \\
\hline B & & $\mathrm{DB}, \mathrm{M}, \mathrm{H}$ & $\mathrm{O} / \mathrm{W}$ & $\mathrm{x}$ & + & + & + & $\mathrm{DB}, \mathrm{M}, \mathrm{H}$ & $\mathrm{O} / \mathrm{W}$ & $\mathrm{x}$ & + & + & + \\
\hline $\mathrm{C}$ & & $\mathrm{DB}, \mathrm{M}, \mathrm{H}$ & $\mathrm{O} / \mathrm{W}$ & $\mathrm{x}$ & + & + & + & $\mathrm{DB}, \mathrm{M}, \mathrm{H}$ & $\mathrm{O} / \mathrm{W}$ & $\mathrm{x}$ & + & + & + \\
\hline
\end{tabular}

*O: organoleptic; LB: light brown; DB: dark brown; M: musk; H: homogen; ET: emulsion type; O/W: oil in water; C: centrifuge; x: no separation; (+ : no change; - : change); $n=3$

Table 4. Spreadability value of formulas before and after irradiation evaluated after $24 \mathrm{~h}$ of formulation and at $90^{\text {th }}$ day of analyses.

\begin{tabular}{|c|c|c|c|c|c|c|c|c|c|}
\hline \multirow{3}{*}{$\begin{array}{l}\mathrm{C} \\
\mathrm{o} \\
\mathrm{d} \\
\mathrm{e}\end{array}$} & \multirow{3}{*}{$\begin{array}{l}\text { Storage } \\
\text { conditions }\end{array}$} & \multicolumn{4}{|c|}{$24 \mathrm{~h}$ after formulation } & \multicolumn{4}{|c|}{$90^{\text {th }}$ day of analyses } \\
\hline & & \multirow{2}{*}{ Unirradiated } & \multicolumn{3}{|c|}{ Dose (kGy) } & \multirow{2}{*}{ Unirradiated } & \multicolumn{3}{|c|}{ Dose (kGy) } \\
\hline & & & 5 & 7.5 & 10 & & 5 & 7.5 & 10 \\
\hline A & $\begin{array}{c}\text { Room } \\
\text { temperature } \\
30 \pm 2^{\circ} \mathrm{C}\end{array}$ & $\begin{array}{l}5787.91 \\
\pm 34.61\end{array}$ & $\begin{array}{l}5429.79 \\
\pm 77.77\end{array}$ & $\begin{array}{l}5061.85 \\
\pm 53.83\end{array}$ & $\begin{array}{l}4394.37 \\
\pm 90.55\end{array}$ & $\begin{array}{l}6351.52 \\
\pm 56.11\end{array}$ & $\begin{array}{c}6097.5 \\
1 \pm 34.83\end{array}$ & $\begin{array}{l}5767.75 \\
\pm 52.31\end{array}$ & $\begin{array}{l}5091.43 \\
\pm 82.46\end{array}$ \\
\hline B & & $\begin{array}{l}6093.10 \\
\pm 93.52\end{array}$ & $\begin{array}{l}5763.30 \\
\pm 63.92\end{array}$ & $\begin{array}{l}5256.98 \\
\pm 49.85\end{array}$ & $\begin{array}{l}4616.14 \\
\pm 46.01\end{array}$ & $\begin{array}{l}6625.07 \\
\pm 56.06\end{array}$ & $\begin{array}{l}6325.69 \\
\pm 67.67\end{array}$ & $\begin{array}{l}5962.26 \\
\pm 55.95\end{array}$ & $\begin{array}{l}5308.47 \\
\pm 38.92\end{array}$ \\
\hline $\mathrm{C}$ & & $\begin{array}{l}6349.16 \\
\pm 53.96\end{array}$ & $\begin{array}{l}6056.18 \\
\pm 72.41\end{array}$ & $\begin{array}{c}5618.75 \\
\pm 112.90\end{array}$ & $\begin{array}{l}4891.20 \\
\pm 90.36\end{array}$ & $\begin{array}{l}7064.79 \\
\pm 41.02\end{array}$ & $\begin{array}{l}6627.48 \\
\pm 57.87\end{array}$ & $\begin{array}{c}6316.52 \\
\pm 113.11\end{array}$ & $\begin{array}{l}5523.69 \\
\pm 59.83\end{array}$ \\
\hline A & & $\begin{array}{l}6062.99 \\
\pm 44.88\end{array}$ & $\begin{array}{l}5772.55 \\
\pm 117.69\end{array}$ & $\begin{array}{l}5519.51 \\
\pm 101.50\end{array}$ & $\begin{array}{c}4753.90 \\
\pm 104.76\end{array}$ & $\begin{array}{l}6685.31 \\
\pm 58.52\end{array}$ & $\begin{array}{l}6401.05 \\
\pm 57.98\end{array}$ & $\begin{array}{l}6095.27 \\
\pm 59.60\end{array}$ & $\begin{array}{l}5497.55 \\
\pm 95.26\end{array}$ \\
\hline B & $\begin{array}{c}\text { High } \\
\text { temperature } \\
40 \pm 2^{\circ} \mathrm{C}\end{array}$ & $\begin{array}{l}6375.05 \\
\pm 46.03\end{array}$ & $\begin{array}{l}6130.03 \\
\pm 91.49\end{array}$ & $\begin{array}{l}5774.49 \\
\pm 57.27\end{array}$ & $\begin{array}{l}5011.48 \\
\pm 28.73\end{array}$ & $\begin{array}{l}7284.97 \\
\pm 58.69\end{array}$ & $\begin{array}{l}6818.79 \\
\pm 59.84\end{array}$ & $\begin{array}{l}6377.40 \\
\pm 38.96\end{array}$ & $\begin{array}{l}5828.44 \\
\pm 44.02\end{array}$ \\
\hline $\mathrm{C}$ & & $\begin{array}{l}6968.39 \\
\pm 68.66\end{array}$ & $\begin{array}{r}6639.94 \\
\pm 141.53\end{array}$ & $\begin{array}{r}6169.66 \\
\pm 132.92\end{array}$ & $\begin{array}{r}5244.40 \\
\pm 102.60\end{array}$ & $\begin{array}{l}7722.40 \\
\pm 66.15\end{array}$ & $\begin{array}{r}7297.83 \\
\pm 121.15\end{array}$ & $\begin{array}{l}6943.70 \\
\pm 48.39\end{array}$ & $\begin{array}{l}6113.70 \\
\pm 52.32\end{array}$ \\
\hline
\end{tabular}

*values were represented as mean \pm standard deviation, $n=3$

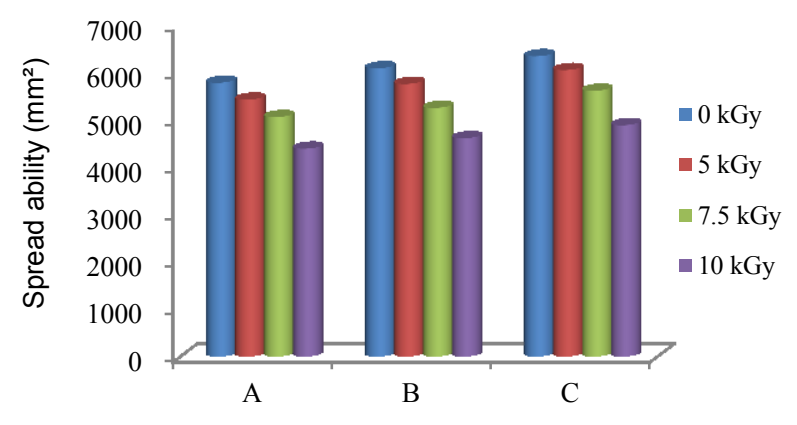

Fig. 3. Spreadability value of formulas before and after irradiation $(n=3)$.

Viscosity value of unirradiated and irradiated formulas were summarized in Table 5. It was determined that increasing extract concentrations caused decreasing viscosity values, while increasing doses of irradiation caused increasing viscosity values as shown in Fig. 4. Changes in viscosity value of all formulas seem depends on extract concentrations $(p<0.05)$ and irradiation dose levels $(p<0.05)$. Apparent viscosity of formulas resulted in decreased values when stored at high temperature and during 90 days of storage.

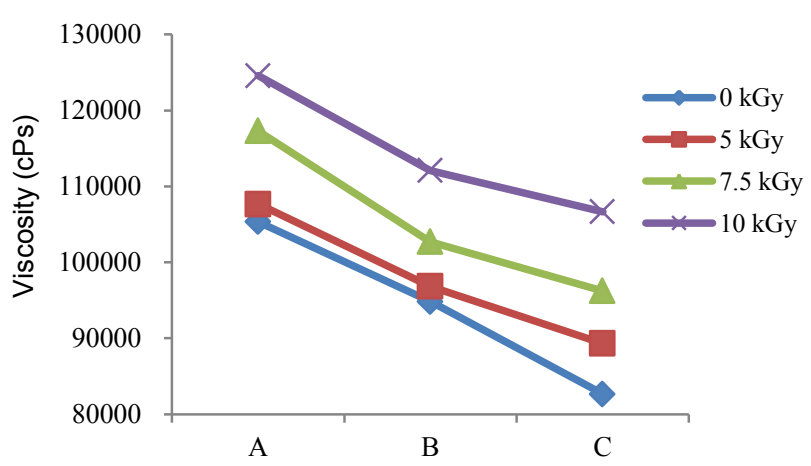

Fig. 4. Viscosity value of formulas before and after irradiation $(n=3)$. 
Table 5. Viscosity value of formulas before and after irradiation evaluated after $24 \mathrm{~h}$ of formulation and at $90^{\text {th }}$ day of analyses.

\begin{tabular}{|c|c|c|c|c|c|c|c|c|c|}
\hline \multirow{3}{*}{$\begin{array}{l}\mathrm{C} \\
\mathrm{o} \\
\mathrm{d} \\
\mathrm{e}\end{array}$} & \multirow{3}{*}{$\begin{array}{c}\text { Storage } \\
\text { conditions }\end{array}$} & \multicolumn{4}{|c|}{$24 \mathrm{~h}$ after formulation } & \multicolumn{4}{|c|}{$90^{\text {th }}$ day of analyses } \\
\hline & & \multirow{2}{*}{ Unirradiated } & \multicolumn{3}{|c|}{ Dose (kGy) } & \multirow{2}{*}{ Unirradiated } & \multicolumn{3}{|c|}{ Dose (kGy) } \\
\hline & & & 5 & 7.5 & 10 & & 5 & 7.5 & 10 \\
\hline A & & $\begin{array}{l}105333.33 \\
\pm 1755.94\end{array}$ & $\begin{array}{l}107666.67 \\
\pm 3253.20\end{array}$ & $\begin{array}{l}117291.67 \\
\pm 1572.88\end{array}$ & $\begin{array}{l}124583.33 \\
\pm 3145.76\end{array}$ & $\begin{array}{l}94833.33 \\
\pm 2254.63\end{array}$ & $\begin{array}{r}100166.67 \\
\pm 3253.20\end{array}$ & $\begin{array}{r}105333.33 \\
\pm 3253.20\end{array}$ & $\begin{array}{l}115833.33 \\
\pm 2602.08\end{array}$ \\
\hline B & $\begin{array}{c}\text { Room } \\
\text { temperature } \\
30 \pm 2^{\circ} \mathrm{C}\end{array}$ & $\begin{array}{c}94833.33 \\
\pm 3013.86\end{array}$ & $\begin{array}{l}96833.33 \\
\pm 2753.79\end{array}$ & $\begin{array}{c}102708.33 \\
\pm 954.70\end{array}$ & $\begin{array}{l}112083.33 \\
\pm 1909.41\end{array}$ & $\begin{array}{c}87333.33 \\
\pm 2843.12\end{array}$ & $\begin{array}{l}92000.00 \\
\pm 3968.63\end{array}$ & $\begin{array}{l}96333.33 \\
\pm 1755.94\end{array}$ & $\begin{array}{l}108958.33 \\
\pm 3207.25\end{array}$ \\
\hline $\mathrm{C}$ & & $\begin{array}{c}82666.67 \\
\pm 2565.80\end{array}$ & $\begin{array}{l}89333.33 \\
\pm 1607.28\end{array}$ & $\begin{array}{c}96250.00 \\
\pm 4098.40\end{array}$ & $\begin{array}{l}106666.67 \\
\pm 3442.23\end{array}$ & $\begin{array}{c}77666.67 \\
\pm 2565.80\end{array}$ & $\begin{array}{c}83833.33 \\
\pm 3013.86\end{array}$ & $\begin{array}{l}88666.67 \\
\pm 1892.97\end{array}$ & $\begin{array}{l}98500.00 \\
\pm 2500.00\end{array}$ \\
\hline A & & $\begin{array}{r}79666.70 \\
\pm 1892.97\end{array}$ & $\begin{array}{l}84666.67 \\
\pm 3253.20\end{array}$ & $\begin{array}{r}90625.00 \\
\pm 2724.31\end{array}$ & $\begin{array}{l}102291.67 \\
\pm 3145.76\end{array}$ & $\begin{array}{c}70666.67 \\
\pm 2565.80\end{array}$ & $\begin{array}{l}76833.33 \\
\pm 2843.12\end{array}$ & $\begin{array}{l}82166.67 \\
\pm 3752.78\end{array}$ & $\begin{array}{l}95208.33 \\
\pm 4434.12\end{array}$ \\
\hline B & $\begin{array}{c}\text { High } \\
\text { temperature } \\
40 \pm 2^{\circ} \mathrm{C}\end{array}$ & $\begin{array}{c}67666.67 \\
\pm 2565.80\end{array}$ & $\begin{array}{l}78000.00 \\
\pm 1322.88\end{array}$ & $\begin{array}{r}79375.00 \\
\pm 2864.11\end{array}$ & $\begin{array}{l}97291.67 \\
\pm 954.70\end{array}$ & $\begin{array}{l}59666.67 \\
\pm 2753.79\end{array}$ & $\begin{array}{l}65666.67 \\
\pm 3253.20\end{array}$ & $\begin{array}{l}73166.67 \\
\pm 2753.79\end{array}$ & $\begin{array}{l}87916.67 \\
\pm 2194.93\end{array}$ \\
\hline $\mathrm{C}$ & & $\begin{array}{c}63833.33 \\
\pm 1258.31\end{array}$ & $\begin{array}{l}67833.33 \\
\pm 1258.31\end{array}$ & $\begin{array}{l}75416.67 \\
\pm 2818.28\end{array}$ & $\begin{array}{l}84583.30 \\
\pm 3207.25\end{array}$ & $\begin{array}{l}54333.33 \\
\pm 2254.63\end{array}$ & $\begin{array}{l}60333.33 \\
\pm 1258.31\end{array}$ & $\begin{array}{l}66000.00 \\
\pm 3278.72\end{array}$ & $\begin{array}{l}75333.33 \\
\pm 3685.56\end{array}$ \\
\hline
\end{tabular}

*values were represented as mean \pm standard deviation, $n=3$

Table 6. Mean particle size value of formulas before and after irradiation evaluated after $24 \mathrm{~h}$ of formulation and at $90^{\text {th }}$ day of analyses.

\begin{tabular}{|c|c|c|c|c|c|c|c|c|c|}
\hline \multirow{3}{*}{$\begin{array}{l}\mathrm{C} \\
\mathrm{o} \\
\mathrm{d} \\
\mathrm{e}\end{array}$} & \multirow{3}{*}{$\begin{array}{l}\text { Storage } \\
\text { conditions }\end{array}$} & \multicolumn{4}{|c|}{$24 \mathrm{~h}$ after formulation } & \multicolumn{4}{|c|}{$90^{\text {th }}$ day of analyses } \\
\hline & & \multirow{2}{*}{ Unirradiated } & \multicolumn{3}{|c|}{ Dose (kGy) } & \multirow{2}{*}{ Unirradiated } & \multicolumn{3}{|c|}{ Dose (kGy) } \\
\hline & & & 5 & 7.5 & 10 & & 5 & 7.5 & 10 \\
\hline A & & $81.46 \pm 0.43$ & $\begin{array}{l}62.00 \\
\pm 0.51\end{array}$ & $\begin{array}{l}55.64 \\
\pm 0.31\end{array}$ & $\begin{array}{l}42.39 \\
\pm 0.31\end{array}$ & $83.51 \pm 0.37$ & $\begin{array}{l}65.35 \\
\pm 0.51\end{array}$ & $\begin{array}{l}59.41 \\
\pm 0.42\end{array}$ & $\begin{array}{l}47.98 \\
\pm 0.65\end{array}$ \\
\hline B & $\begin{array}{c}\text { Room } \\
\text { temperature } \\
30 \pm 2^{\circ} \mathrm{C}\end{array}$ & $83.13 \pm 0.21$ & $\begin{array}{c}63.79 \\
\pm 0.26\end{array}$ & $\begin{array}{l}56.69 \\
\pm 0.35\end{array}$ & $\begin{array}{l}43.62 \\
\pm 0.36\end{array}$ & $85.66 \pm 0.37$ & $\begin{array}{r}67.67 \\
\pm 0.31\end{array}$ & $\begin{array}{c}61.61 \\
\pm 0.34\end{array}$ & $\begin{array}{r}49.35 \\
\pm 0.34\end{array}$ \\
\hline $\mathrm{C}$ & & $84.43 \pm 0.47$ & $\begin{array}{l}64.70 \\
\pm 0.25\end{array}$ & $\begin{array}{c}58.53 \\
\pm 0.42\end{array}$ & $\begin{array}{l}44.33 \\
\pm 0.50\end{array}$ & $87.56 \pm 0.29$ & $\begin{array}{c}69.82 \\
\pm 0.33\end{array}$ & $\begin{array}{c}63.73 \\
\pm 0.26\end{array}$ & $\begin{array}{l}52.57 \\
\pm 0.45\end{array}$ \\
\hline A & & $82.58 \pm 0.37$ & $\begin{array}{c}63.95 \\
\pm 0.44\end{array}$ & $\begin{array}{l}56.85 \\
\pm 0.29\end{array}$ & $\begin{array}{l}45.31 \\
\pm 0.26\end{array}$ & $87.53 \pm 0.72$ & $\begin{array}{c}68.07 \\
\pm 0.46\end{array}$ & $\begin{array}{c}62.71 \\
\pm 0.33\end{array}$ & $\begin{array}{l}51.01 \\
\pm 0.31\end{array}$ \\
\hline B & $\begin{array}{c}\text { High } \\
\text { temperature } \\
40 \pm 2^{\circ} \mathrm{C}\end{array}$ & $84.94 \pm 0.37$ & $\begin{array}{l}65.58 \\
\pm 0.43\end{array}$ & $\begin{array}{c}58.98 \\
\pm 0.32\end{array}$ & $\begin{array}{l}46.34 \\
\pm 0.36\end{array}$ & $90.44 \pm 0.45$ & $\begin{array}{c}70.05 \\
\pm 0.42\end{array}$ & $\begin{array}{c}63.92 \\
\pm 0.39\end{array}$ & $\begin{array}{l}53.28 \\
\pm 0.28\end{array}$ \\
\hline $\mathrm{C}$ & & $86.77 \pm 0.38$ & $\begin{array}{l}67.25 \\
\pm 0.39\end{array}$ & $\begin{array}{c}60.68 \\
\pm 0.35\end{array}$ & $\begin{array}{l}47.42 \\
\pm 0.28\end{array}$ & $91.71 \pm 0.57$ & $\begin{array}{l}72.38 \\
\pm 0.35\end{array}$ & $\begin{array}{c}66.57 \\
\pm 0.43\end{array}$ & $\begin{array}{l}55.65 \\
\pm 0.29\end{array}$ \\
\hline
\end{tabular}

*values were represented as mean \pm standard deviation, $n=3$

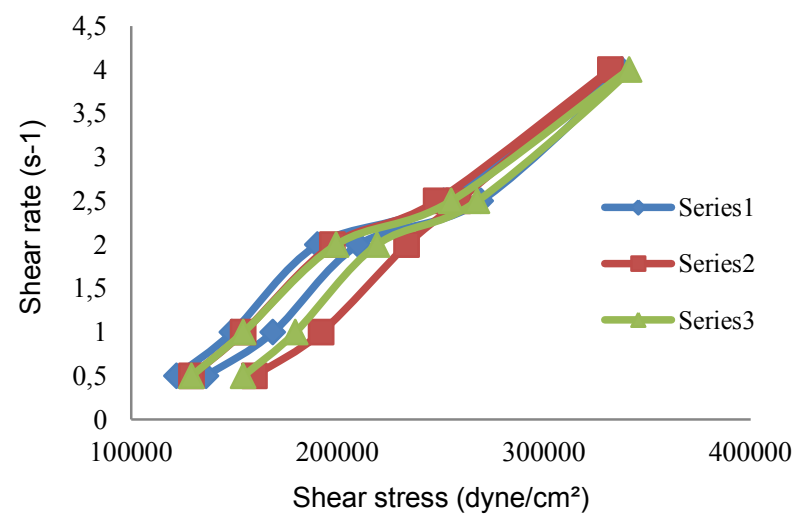

Fig. 5. Rheogram of formula A after irradiation dose of $7.5 \mathrm{kGy}$ at 90 days of analyses $(n=3)$.
Figure 5 showed that formula A had thixotropic plastic behaviour. These result represented that the formula did not begin to flow until a shearing stress, corresponding to the yield value, was exceeded. At moderately high shear rate, formulation was easy to apply to the skin.

Mean particle size value of unirradiated and irradiated formulas were summarized in Table 6 . It was determined that increasing extract concentrations caused increasing particle size values, while increasing doses of irradiation caused decreasing particle size values as shown at Fig. 6. Changes in mean particle size value of all formulas seem depends on extract concentrations $(p<0.05)$ 
and irradiation dose levels $(\mathrm{p}<0.05)$. Apparent mean particle size of formulas resulted in increased values when stored at high temperature and during 90 days of storage.

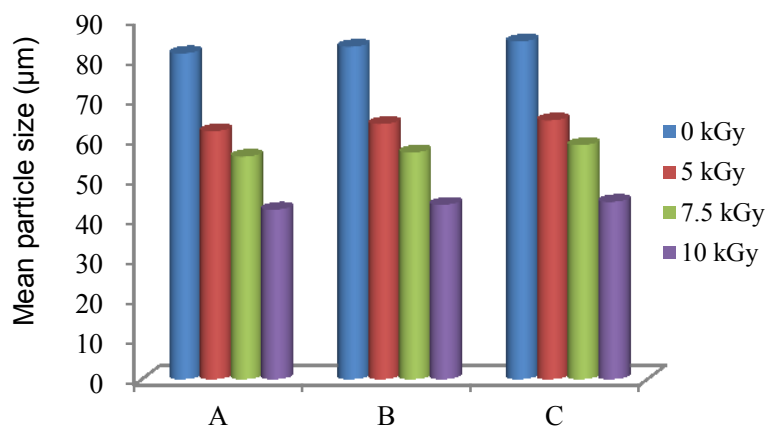

Fig. 6. Mean particle size value of formulas before and after irradiation $(n=3)$.

The presence of G. mangostana pericarp extract at the formulas affected their particle size values, due to the increasing of water soluble components at the dispersing phase from the bioactive subtances such as tanin, flavonoid, alkaloid in G. mangostana pericarp extract. Increasing of the dispersing phase was not followed by emulgator enhancement in formula, thus led to insufficient in an interfacial film between the dispersed and dispersing phase, the dispersing phase tended to form larger droplets, that caused decreasing in viscosity values. The lower the viscosity value is, the ability to spread is also getting lower. At higher temperature condition $\left(40 \pm 2{ }^{\circ} \mathrm{C}\right)$, the particle size values had increased due to the effect of the elevated temperature of storage which caused an acceleration of the motion rate of the dispersed phase.

$\mathrm{pH}$ value of unirradiated and irradiated formulas were summarized in Table 7. It was determined that increasing extract concentrations and doses of irradiation caused decreasing $\mathrm{pH}$ values, probably, due to the acidity of the extract, as shown in Fig. 7. Changes in $\mathrm{pH}$ value of all formulas seem depends on extract concentrations $(p<0.05)$ and irradiation dose levels $(\mathrm{p}<0.05)$. The formulas presented tendency to reduce the $\mathrm{pH}$ value at high temperature and during 90 days of storage condition, probably, due to the deterioration of the components of the fluid emulsions and the bioactive substances from the extract of $G$. mangostana.

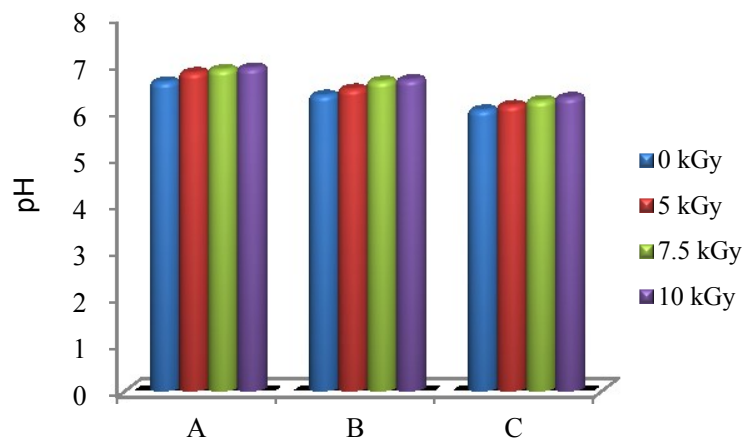

Fig. 7. $\mathrm{pH}$ value of formulas before and after irradiation $(n=3)$.

\section{Microbiological properties}

The microbial contamination value of unirradiated and irradiated formulas were summarized in Table 8. An irradiation dose of $5 \mathrm{kGy}$ on formulas could eliminate mold-yeast from initial contamination while to remove bacterial contamination completely, the higher dose is needed. Bacterial contamination can be removed completely by irradiation dose of $\geq 7.5 \mathrm{kGy}$. Changes in microbial contamination of all formulas seem not depends on extract concentrations $(p>0.05)$ and depends on irradiation dose levels $(p<0.05)$. High temperature storage condition did not affected total microbial contamination value significantly during 90 days of storage.

Table 7. PH value of formulas before and after irradiation evaluated after $24 \mathrm{~h}$ of formulation and at $90^{\text {th }}$ day of analyses.

\begin{tabular}{|c|c|c|c|c|c|c|c|c|c|}
\hline \multirow{3}{*}{$\begin{array}{l}\mathrm{C} \\
\mathrm{o} \\
\mathrm{d} \\
\mathrm{e}\end{array}$} & \multirow{3}{*}{$\begin{array}{l}\text { Storage } \\
\text { conditions }\end{array}$} & \multicolumn{4}{|c|}{$24 \mathrm{~h}$ after formulation } & \multicolumn{4}{|c|}{$90^{\text {th }}$ day of analyses } \\
\hline & & \multirow{2}{*}{ Unirradiated } & \multicolumn{3}{|c|}{ Dose (kGy) } & \multirow{2}{*}{ Unirradiated } & \multicolumn{3}{|c|}{ Dose (kGy) } \\
\hline & & & 5 & 7.5 & 10 & & 5 & 7.5 & 10 \\
\hline A & \multirow{3}{*}{$\begin{array}{c}\text { Room } \\
\text { temperature } \\
30 \pm 2{ }^{\circ} \mathrm{C}\end{array}$} & $6.62 \pm 0.03$ & $6.82 \pm 0.03$ & $6.88 \pm 0.06$ & $6.92 \pm 0.03$ & & $6.28 \pm 0.03$ & $6.27 \pm 0.06$ & $6.28 \pm 0.03$ \\
\hline B & & $6.33 \pm 0.03$ & $6.47 \pm 0.03$ & $6.63 \pm 0.03$ & $6.67 \pm 0.03$ & $6.17 \pm 0.03$ & $6.12 \pm 0.06$ & $6.10 \pm 0.05$ & $6.02 \pm 0.03$ \\
\hline $\mathrm{C}$ & & $6.02 \pm 0.03$ & $6.12 \pm 0.03$ & $6.22 \pm 0.03$ & $6.28 \pm 0.03$ & $5.77 \pm 0.06$ & $5.68 \pm 0.03$ & $5.67 \pm 0.06$ & $5.67 \pm 0.00$ \\
\hline A & \multirow{3}{*}{$\begin{array}{c}\text { High } \\
\text { temperature } \\
40 \pm 2^{\circ} \mathrm{C}\end{array}$} & $6.53 \pm 0.03$ & $6.67 \pm 0.06$ & $6.72 \pm 0.03$ & $6.82 \pm 0.03$ & $6.17 \pm 0.03$ & $6.03 \pm 0.03$ & $5.97 \pm 0.06$ & $5.83 \pm 0.06$ \\
\hline B & & $6.17 \pm 0.03$ & $6.27 \pm 0.06$ & $6.38 \pm 0.03$ & $6.48 \pm 0.03$ & $5.68 \pm 0.03$ & $5.58 \pm 0.03$ & $5.53 \pm 0.03$ & $5.47 \pm 0.03$ \\
\hline $\mathrm{C}$ & & $5.82 \pm 0.03$ & $6.02 \pm 0.03$ & $6.07 \pm 0.06$ & $6.12 \pm 0.03$ & $5.48 \pm 0.06$ & $5.38 \pm 0.06$ & $5.48 \pm 0.03$ & $5.33 \pm 0.06$ \\
\hline
\end{tabular}

*values were represented as mean \pm standard deviation, $n=3$ 
Table 8. Microbial contamination value of formulas before and after irradiation after $24 \mathrm{~h}$ of formulation and at $90^{\text {th }}$ day of analyses.

\begin{tabular}{|c|c|c|c|c|c|c|c|c|c|c|}
\hline \multirow{3}{*}{$\begin{array}{l}\mathrm{C} \\
\mathrm{o} \\
\mathrm{d} \\
\mathrm{e}\end{array}$} & \multirow{3}{*}{$\begin{array}{c}\text { Storage } \\
\text { conditions }\end{array}$} & \multirow{3}{*}{$\mathrm{MC}$} & \multicolumn{4}{|c|}{$24 \mathrm{~h}$ after formulation } & \multicolumn{4}{|c|}{$90^{\text {th }}$ day of analyses } \\
\hline & & & \multirow{2}{*}{ Unirradiated } & \multicolumn{3}{|c|}{ Dose (kGy) } & \multirow{2}{*}{ Unirradiated } & \multicolumn{3}{|c|}{ Dose (kGy) } \\
\hline & & & & 5 & 7.5 & 10 & & 5 & 7.5 & 10 \\
\hline \multirow{2}{*}{ A } & \multirow{6}{*}{$\begin{array}{c}\text { Room } \\
\text { temperature } \\
30 \pm 2^{\circ} \mathrm{C}\end{array}$} & $\mathrm{B}$ & $3.55 \times 10^{4}$ & $3.15 \times 10^{3}$ & 0 & 0 & $2.14 \times 10^{7}$ & $3.31 \times 10^{5}$ & 0 & 0 \\
\hline & & MY & $3.13 \times 10^{3}$ & 0 & 0 & 0 & $3.52 \times 10^{5}$ & 0 & 0 & 0 \\
\hline \multirow{2}{*}{ B } & & B & $4.09 \times 10^{4}$ & $4.09 \times 10^{3}$ & 0 & 0 & $2.55 \times 10^{7}$ & $3.88 \times 10^{5}$ & 0 & 0 \\
\hline & & MY & $3.83 \times 10^{3}$ & 0 & 0 & 0 & $4.34 \times 10^{5}$ & 0 & 0 & 0 \\
\hline \multirow{2}{*}{$\mathrm{C}$} & & $\mathrm{B}$ & $4.67 \times 10^{4}$ & $5.08 \times 10^{3}$ & 0 & 0 & $3.02 \times 10^{7}$ & $4.39 \times 10^{5}$ & 0 & 0 \\
\hline & & MY & $4.67 \times 10^{3}$ & 0 & 0 & 0 & $4.86 \times 10^{5}$ & 0 & 0 & 0 \\
\hline \multirow{2}{*}{ A } & \multirow{6}{*}{$\begin{array}{c}\text { High } \\
\text { temperature } \\
40 \pm 2^{\circ} \mathrm{C}\end{array}$} & B & $4.15 \times 10^{4}$ & $3.92 \times 10^{3}$ & 0 & 0 & $2.50 \times 10^{7}$ & $4.08 \times 10^{5}$ & 0 & 0 \\
\hline & & MY & $3.40 \times 10^{3}$ & 0 & 0 & 0 & $3.40 \times 10^{5}$ & 0 & 0 & 0 \\
\hline \multirow{2}{*}{ B } & & B & $4.58 \times 10^{4}$ & $4.82 \times 10^{3}$ & 0 & 0 & $2.93 \times 10^{7}$ & $4.82 \times 10^{5}$ & 0 & 0 \\
\hline & & MY & $3.95 \times 10^{3}$ & 0 & 0 & 0 & $3.98 \times 10^{5}$ & 0 & 0 & 0 \\
\hline \multirow{2}{*}{$\mathrm{C}$} & & $\mathrm{B}$ & $5.51 \times 10^{4}$ & $5.96 \times 10^{3}$ & 0 & 0 & $3.42 \times 10^{7}$ & $5.59 \times 10^{5}$ & 0 & 0 \\
\hline & & MY & $4.74 \times 10^{3}$ & 0 & 0 & 0 & $4.60 \times 10^{5}$ & 0 & 0 & 0 \\
\hline
\end{tabular}

*MC: microbial contamination (cfu/g); B: bacteria; MY: mold-yeast; values were represented as mean \pm standard deviation, $n=3$

Table 9. $\mathrm{R}_{f}$ values and densitogram peak areas of formula A before and after irradiation after $24 \mathrm{~h}$ of formulation and at $90^{\text {th }}$ day of analyses $(\lambda=317 \mathrm{~nm})$.

\begin{tabular}{|c|c|c|c|c|c|c|c|c|c|}
\hline \multirow{3}{*}{ Peak } & \multirow{3}{*}{$\mathrm{R}_{f}$} & \multicolumn{4}{|c|}{$24 \mathrm{~h}$ after formulation } & \multicolumn{4}{|c|}{$90^{\text {th }}$ day of analyses } \\
\hline & & \multirow{2}{*}{ Unirradiated } & \multicolumn{3}{|c|}{ Dose (kGy) } & \multirow{2}{*}{ Unirradiated } & \multicolumn{3}{|c|}{ Dose (kGy) } \\
\hline & & & 5 & 7.5 & 10 & & 5 & 7.5 & 10 \\
\hline $1^{\text {st }}$ & $0.35-0.40$ & 1777.8 & 2040.2 & 2564.5 & 3036.5 & 1237.8 & 1831.7 & 2492.7 & 3005.2 \\
\hline $2^{\text {nd }}$ & $0.42-0.47$ & 4527.4 & 5254.8 & 5854.1 & 6016.5 & 4045.3 & 4864.5 & 5802.3 & 5968.6 \\
\hline $3^{\text {rd }}$ & $0.47-0.56$ & 2854.1 & 2901 & 3061.1 & 4098.3 & 2451.3 & 2603.9 & 2998.2 & 4052.8 \\
\hline $4^{\text {th }}$ & $0.62-0.72$ & 16032.8 & 17858.1 & 19239.3 & 20587.5 & 14325.8 & 16622.6 & 19094.6 & 20462.4 \\
\hline $5^{\text {th }}$ & $0.80-0.95$ & 9231.9 & 12177.1 & 13625.1 & 14600.4 & 8101.9 & 11467.8 & 13394.8 & 14473.6 \\
\hline
\end{tabular}

\section{TLC-densitometry on formulas}

Based on the densitogram 3D profiles $(\lambda=317 \mathrm{~nm})$ there were at least 5 peak areas observed in formula A before and after irradiation (Fig. 8).

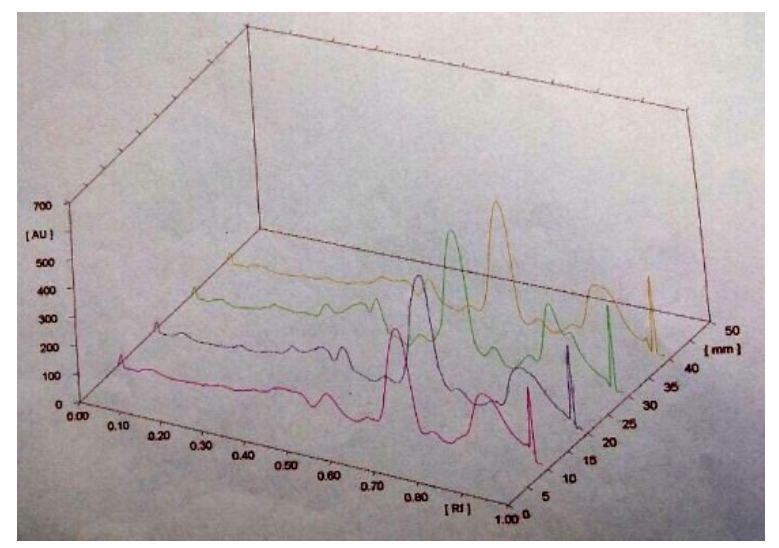

Fig. 8. Densitogram 3D profiles of unirradiated and irradiated formula $A$ at 90 days of analyses $(\lambda=317 \mathrm{~nm})$.
Table 9 shows that there were an alteration between unirradiated and irradiated peak areas. The presence of peak area alterations allegedly due to the effects of irradiation. Generally, it is shown that irradiation dose up to $10 \mathrm{kGy}$ caused increasing of peak area. The increasing of peak area possible due to the effects of irradiation that caused the formation of new components. However, there were also decreasing in peak areas, which is possibly caused by degradation of the components into the other components.

\section{CONCLUSION}

The use of gamma irradiation for decontamination is advantageous for final cosmetic products as well as raw materials. Sterilization is not an obligation for cosmetic products. However, they have to be protected from any contamination or deterioration. The G. mangsotana pericarp extract cream formulas with irradiation dose until 
$10 \mathrm{kGy}$ presented acceptable physicochemical and microbiological stabilities, for at least 90 days, when the formulas were stored at room temperature $\left(30 \pm 2^{\circ} \mathrm{C}\right)$ and at high temperature $\left(40 \pm 2^{\circ} \mathrm{C}\right)$. Formula A which contained $1 \%$ of $G$. mangostana pericarp extract with irradiation dose of $7.5 \mathrm{kGy}$ is the optimum formula from this research.

\section{ACKNOWLEDGMENT}

The authors wish to express our gratitude for facilities and supports from Pancasila University and Center for Application of Isotopes and Radiation Technology, National Nuclear Energy Agency, Indonesia.

\section{REFERENCES}

1. T. Mitsui, New Cosmetic Science, Elsevier, New York (1997) 38.

2. M. Morariu, Topical Formulations and Method of Use, US Patent 0216251A1 (2006).

3. R.I. Tranggono and F. Latifah, Handbook of Cosmetics, Gramedia, Jakarta (2007) 30. (In Indonesian).

4. E. Mardawati, C.S. Achyar and H. Marta, Study of antioxidant activity of mangosteen pericarp extract (Garcinia mangostana L.) in order to use the waste of mangosteen pericarp in regency of Puspahiang, district of Tasikmalaya, Faculty of Agriculture Industry Technology - Padjajaran University, Bandung (2008). (In Indonesian).
5. A.E. Nugroho, Mangosteen (Garcinia mangostana L.) from the wasted pericarp into a candidate for a drug, Faculty of Pharmacy Gadjah Mada University, Jogyakarta (2012). (In Indonesian).

6. C.N. Gupita and A. Rahayuni, Journal of Nutrition College (2012). (In Indonesian).

7. J.W. Wadsworth, S.P. Story, B. Zhou, et al., Garcinia mangostana L. Enhanced Animal Food Product, US Patent 7244463 B2 (2007).

8. N. Ochiai, S. Inomata and K. Takada, Method for Pressuring Reduction of Elasticity of Skin, US Patent 0077523A1 (2004).

9. Anonymous, Legislation on Traditional Herbal Medicines, Directorate General of Food and Drug Administration, Department of Health of the Republic of Indonesia, Jakarta (1999). (In Indonesian).

10. R.C. Rowe, P.J. Sheskey and M.E. Quinn, Handbook of Pharmaceutical Excipients, $6^{\text {th }}$ ed., Pharmaceutical Press, London (2009).

11. R. Chosdu, Darmawan and Erizal, The influence of gamma irradiation on the quality of baby cosmetics, BATAN, Jakarta (1996). (In Indonesian).

12. Anonymous, Standard Method Test for Microbe Contaminants, The Agency of National Standard (BSN), Jakarta, SNI 01-2897 (1992). (In Indonesian).

13. H.A. Jung, B.N. Su, W.J. Keller, et al., J. Agric. Food Chem. (2006). 